\title{
Passive control of flexible structures by confinement of vibrations
}

\author{
M. Ouled Chtiba ${ }^{\mathrm{a}}$, S. Choura ${ }^{\mathrm{a}}$, S. El-Borgi ${ }^{\mathrm{a}}$ and A.H. Nayfeh ${ }^{\mathrm{b}, *}$ \\ ${ }^{a}$ Applied Mechanics Research Laboratory, Tunisia Polytechnic School, La Marsa, Tunisia \\ ${ }^{\mathrm{b}}$ Department of Engineering Sciences and Mechanics, Virginia Tech, Blacksburg, VA, USA
}

Received 26 April 2006

\begin{abstract}
We propose a two-step strategy for the design of passive controllers for the simultaneous confinement and suppression of vibrations (SCSV) in mechanical structures. Once the sensitive and insensitive elements of these structures are identified, the first design step synthesizes an active control law, which is referred to as the reference control law (RCL), for the SCSV. We show that the problem of SCSV can be formulated as an LQR-optimal control problem through which the maximum amplitudes, associated with the control input and the displacements of the sensitive and insensitive parts, can be regulated. In the second design step, a transformation technique that yields an equivalent passive controller is used. Such a technique uses the square root of sum of squares method to approximate an equivalent passive controller while maximizing the effects of springs and dampers characterizing passive elements that are added to the original structure. The viability of the proposed control design is illustrated using a three-DOF mechanical system subject to an excitation. It is assumed that all of the masses are sensitive to the excitation, and thus the vibratory energy must be confined in the added passive elements (insensitive parts). We show that the vibration amplitudes associated with the sensitive masses are attenuated at fast rate at the expense of slowing down the convergence of the passive elements to their steady states. It is also demonstrated that a combination of the RCL and the equivalent passive control strategy leads to similar structural performance.
\end{abstract}

Keywords: LQR-optimal control, state and input bounds, active control, passive control, confinement of vibrations

\section{Introduction}

In engineering applications, vibrations are likely to excite unwanted resonances in flexible structures. In particular, if these structures have low material damping, the vibratory motion can persist for a long time before it dies out, and thus problems in structural performance may arise. Therefore, it may be of interest to reduce the vibrations in the more sensitive parts of the structure while transferring the vibrational energy to the less sensitive parts. These structures include space satellites equipped with sensitive communications antennas and flexible robot manipulators with sensitive payload masses. The common practice is to place force and torque actuators with appropriate time-varying magnitudes at the span of the structure. These actuators must provide sufficient damping for energy dissipation. The rate of vibration reduction in all parts of the structure is approximately the same; that is, it would take the same time to bring to rest both of the sensitive parts and those that are irrelevant to certain performance specifications. In some cases, it is desirable to suppress the vibrations at a faster rate at those sensitive regions or stations.

Recently, considerable effort has been dedicated to devise effective control designs for flexible structures. There are some researchers, such as Snyder et al. [15] and Bendiksen [1], who addressed the notion of vibration localization (Anderson localization) in flexible structures. Snyder et al. [15] used a one-dimensional system of masses with nearest-neighbor interactions and periodic boundary conditions to study mode decay and ergodicity in nonlinear

\footnotetext{
*Corresponding author. E-mail: anayfeh@vt.edu.
} 
disordered systems. Their results indicate that the rates of mode decay at early times increase when impurities are added. However, for long times this rate decreases with increasing impurity mass and impurity concentrations.

Bendiksen [1] studied the localization phenomenon in engineering structures that include periodic or nearly periodic multi-span beams and trusses, large space structures, and almost periodic structures with circular symmetry, such as bladed disks in turbomachines. He discussed both analytical and numerical methods for analyzing and predicting localization in finite- and infinite-dimensional systems. He showed that localization results from damage in bladed disks of turbomachines. Castanier et al. [3] employed the Lyapunov exponents of the system wave-transfer matrix to analyze localization in multi-coupled, in particular mono-coupled, disordered periodic linear systems. They concluded that the Lyapunov exponents predict the decay rate of two wave types in the absence of energy leakage from one wave type to another. Castanier et al. [2] and Langley [11] discussed localization by damping in periodic and nearly periodic structures. They investigated the effect of damping on the response and noted an increased vibration localization (or concentration near the excitation point) arising from the attenuation factor. Shahruz [21] examined the occurrence of vibration localization in mistuned periodic structures and proposed a passive method to eliminate such localization.

Yigit and Choura [23] and Choura and Yigit [6,7] developed a strategy for active control of vibrations by confinement. Their strategy consists of assigning the eigenstructure (both eigenvalues and eigenvectors) of flexible structures for the purpose of simultaneous confinement and suppression of vibrations where the modal matrix plays a key role in the energy redistribution. Such strategy guarantees both vibration confinement and structural stability. Ouled Chtiba et al. [13] discussed hybrid control of seismically excited structures by vibration confinement. Their control strategy consists of adding bracing elements as the passive and insensitive elements of the structure. Then active controllers are applied to the structure to transfer the vibrational energy from the floors to the bracing elements.

It has been reported that active controllers are not reliable for the mitigation of vibrations in most mechanical and civil engineering structures, because electrically-operated controllers are subject to current failure. Alternatively, several researchers proposed strategies for vibration suppression by adding passive elements, such as masses, springs and dampers, to the structure [10,22]. Choura et al. [8] developed a strategy for passive vibration confinement of a two-degree-of-freedom oscillator. Ribakov et al. [8], Reinhorn et al. [16,17], and Gluck et al. [10] synthesized optimal control laws using LQR. These laws are adopted for the design of linear passive viscous devices according to their deformation and velocity. Gluck et al. [10] demonstrated that the response of a structure dominated by a single mode of vibration and equipped with optimally designed supplemental viscous dampers is similar to that of an actively controlled structure. They claimed that passivity is an important property of dynamic systems, and hence a passive system can be robustly stabilized by any strictly passive controller, despite unmodeled dynamics and parametric uncertainties.

In this study, we show that the problem of vibration confinement can be formulated as an LQR-optimal control problem. This strategy is an alternative to that of Choura [5] for vibration confinement in prespecified regions of the structure along with regulation of its states and the control input. Choi et al. [4] showed that the LQR design regulator is more robust than eigenstructure assignment. We adopt this strategy to design linear passive controllers. The emphasis is on designing a control strategy that adds passive devices (linear springs and dampers) for confining and suppressing simultaneously the vibrational energy in flexible structures. To this end, we first design an optimal controller using a set of actuators to satisfy desired confinement and suppression specifications. Then, we construct an equivalent passive controller, which produces a similar performance.

\section{Problem formulation and objective}

Consider the $n$-dimensional discretized model of flexible structures

$$
M \ddot{x}+C \dot{x}+K x=B_{s} u(t)+E_{s} w(t)
$$

where $x, \dot{x}$ and $\ddot{x}$ are, respectively, the $n \times 1$ displacement, velocity, and acceleration vectors; $M$ is a symmetric positive definite mass matrix; $C$ is the internal damping matrix; $K$ is a symmetric positive stiffness matrix; $u(t)$ is an $n \times 1$ vector of actuator-supplied inputs; $B_{s}$ is the $n \times m$ matrix locating the actuators; and $E_{s}$ is the $n \times 1$ vector associated with the disturbance input $w(t)$. Let $n$ sensors generate the output given by $D x$, where $D$ is a nonsingular 
square matrix of appropriate dimension. Without loss of generality, let $D$ be the identity matrix of appropriate dimension. The objectives of this paper are twofold: (1) synthesize an active control law $u(t)$ for confining and suppressing simultaneously the vibrational energy in flexible structures and regulating both of the states and inputs, and (2) find a set of passive elements that replaces the active controller and produces a similar structural performance.

\section{Strategy of vibration confinement and suppression}

At this stage, we consider the design of an $n$-dimensional optimal feedback control $u(t)$ for the vibration confinement and suppression in the flexible structure described in Eq. (1). More control emphasis will be devoted to the sensitive elements in the sense that their convergence to the desired steady state is attained a fast rate. Consequently, the proposed control strategy not only confines and suppresses the vibrations but also regulates the input and state signals in contrast with control by confinement of vibration strategies. The proposed strategy converts the original vibratory modes into a set of modes that allows the vibrational energy to be confined in prescribed regions of the structural domain. We show that this strategy can be established by formulating the problem of vibration confinement as an LQR problem.

In state-space form, Eq. (1) can be rewritten as

$$
\dot{X}=A X+B u(t)+E w(t)
$$

where

$$
X=\left[\begin{array}{l}
x \\
\dot{x}
\end{array}\right], \dot{X}=\left[\begin{array}{l}
\dot{x} \\
\ddot{x}
\end{array}\right], A=\left[\begin{array}{cc}
0 & I \\
-M^{-1} K-M^{-1} C
\end{array}\right], B=\left[\begin{array}{l}
0 \\
M^{-1} B_{s}
\end{array}\right] \text { and } E=\left[\begin{array}{l}
0 \\
M^{-1} E_{s}
\end{array}\right]
$$

It is commonly known that pole-assignment techniques do not consider external disturbances. Thus, in the absence of disturbances, Eq. (2) becomes

$$
\dot{X}=A X+B u(t)
$$

The design of an observer-based LQR controller involves the determination of optimal control inputs that minimize a quadratic performance function, which reflects the penalty on the states and control energy. The problem of optimal control is given by

$$
J=\frac{1}{2} \int_{0}^{\infty}\left(X^{T} S X+u^{T} R u\right) d t
$$

subject to

$$
\dot{X}=A X+B u(t)
$$

where $S \geqslant 0$ and $R>0$ are, respectively, weighting matrices whose major role is the confinement of the vibrational energy in selected regions of the spatial domain. In particular, the choice of their diagonal elements determines the way in which the vibrational energy is reallocated in the system domain. The force vector is defined by

$$
u(t)=-[G H] X=-L X=-(G x+H \dot{x})
$$

where:

$$
L=R^{-1} B P
$$

is the gain matrix obtained from the minimization of the performance index Eq. (4a) and $P$ is the solution of the matrix Riccati equation

$$
P A+A^{T} P-P B R^{-1} B^{T} P+S=0
$$

Now, the objective is to exploit the optimal solution for the problem of vibration confinement and suppression. To this end, we consider the following linear transformation:

$$
X=U \eta=\left[\begin{array}{ll}
Q & 0 \\
0 & Q
\end{array}\right] \eta
$$


where $Q$ is the confinement matrix defined by Choura [5] and Choura et al. [7]. To guarantee lower energy levels in the sensitive parts, the elements of the rows in $Q$ associated with the sensitive parts should be lower than those associated with the insensitive elements. Substituting Eq. (8) into Eq. (3) yields

$$
\dot{\eta}=A_{\eta} \eta+B_{\eta} u(t)
$$

where $A_{\eta}=U^{-1} A U$ and $B_{\eta}=U^{-1} B$. The optimal control problem can be restated as

$$
J_{\eta}=\frac{1}{2} \int_{0}^{\infty}\left(\eta^{T} S_{\eta} \eta+u^{T} R u\right) d t
$$

subject to

$$
\dot{\eta}=A_{\eta} \eta+B_{\eta} u(t)
$$

where $S_{\eta}$ is the weighting matrix associated with the new variable $\eta$. The following theorem states the condition under which the performance indices $J_{\eta}$ and $J$ are equivalent:

Theorem 1. Given the performance index $J$ associated with the physical system described by Eq. (4) and the performance index associated with the modal dynamics described by Eq. (10) $J_{\eta}$ and $J$ are equivalent if and only if $S=\left(U^{-1}\right)^{T} S_{\eta} U^{-1}$.

Proof: Consider a flexible structure whose dynamics are described by Eq. (1). Using the linear transformation

$$
x=Q \psi
$$

we rewrite Eq. (1) as

$$
\ddot{\psi}+Q^{-1} M^{-1} C Q \dot{\psi}+Q^{-1} M^{-1} K Q \psi=Q^{-1} M^{-1} B_{s} u(t)+Q^{-1} M^{-1} E_{s} w(t)
$$

Let the state space vector be defined as follows:

$$
\eta^{T}=\left[\psi^{T} \dot{\psi}^{T}\right]
$$

Without the external excitation, substituting Eq. (13) into Eq. (12) yields Eq. (9).

We define the Hamiltonian matrices associated, respectively, with the physical and transformed systems as

$$
H=\left[\begin{array}{cc}
A & -B R^{-1} B^{T} \\
-S & -A^{T}
\end{array}\right] \quad H_{\eta}=\left[\begin{array}{cc}
A_{\eta} & -B_{\eta} R^{-1} B_{\eta}^{T} \\
-S_{\eta} & -A_{\eta}^{T}
\end{array}\right]
$$

Let $\Lambda$ be the $4 n$-diagonal matrix whose elements are the eigenvalues of matrix $H_{\eta}$ and $Y$ be the $4 n \times 4 n$ matrix whose columns are the eigenvectors of matrix $H_{\eta}$, then

$$
H_{\eta} Y=Y \Lambda
$$

We decompose $Y$ so that $Y^{T}=\left[Y_{1}^{T} Y_{2}^{T}\right]$ and rewrite Eq. (15) as

$$
\begin{aligned}
& {\left[\begin{array}{l}
A_{\eta}-B_{\eta} R^{-1} B_{\eta}^{T} \\
-S_{\eta}-A_{\eta}^{T}
\end{array}\right]\left[\begin{array}{l}
Y_{1} \\
Y_{2}
\end{array}\right]=\left[\begin{array}{l}
Y_{1} \\
Y_{2}
\end{array}\right] \Lambda} \\
& \left\{\begin{array}{l}
U^{-1} A U Y_{1}-U^{-1} B R^{-1} B^{T}\left(U^{-1}\right)^{T} Y_{2}=Y_{1} \Lambda \\
-S_{\eta} Y_{1}-U^{T} A^{T}\left(U^{-1}\right)^{T} Y_{2}=Y_{2} \Lambda
\end{array}\right. \\
& \left\{\begin{array}{l}
A U Y_{1}-B R^{-1} B^{T}\left(U^{-1}\right)^{T} Y_{2}=U Y_{1} \Lambda \\
-\left(U^{-1}\right)^{T} S_{\eta} U^{-1} U Y_{1}-A^{T}\left(U^{-1}\right)^{T} Y_{2}=\left(U^{-1}\right)^{T} Y_{2} \Lambda
\end{array}\right. \\
& \left\{\begin{array}{l}
A Z_{1}-B R^{-1} B^{T} Z_{2}=Z_{1} \Lambda \\
-\left(U^{-1}\right)^{T} S_{\eta} U^{-1} Z_{1}-A^{T} Z_{2}=Z_{2} \Lambda
\end{array}\right.
\end{aligned}
$$

where $Z_{1}=U Y_{1}$ and $Z_{2}=\left(U^{-1}\right)^{T} Y_{2}$. Equation (19) can be rewritten in the following matrix form: 


$$
\left[\begin{array}{ll}
A & -B R^{-1} B^{T} \\
-\left(U^{-1}\right)^{T} S_{\eta} U^{-1}-A^{T}
\end{array}\right]\left[\begin{array}{l}
Z_{1} \\
Z_{2}
\end{array}\right]=\left[\begin{array}{l}
Z_{1} \\
Z_{2}
\end{array}\right] \Lambda
$$

It follows from Eq. (20) and the Hamiltonian associated with the physical system that

$$
S=\left(U^{-1}\right)^{T} S_{\eta} U^{-1}
$$

For instance, if the weighting matrix $S_{\eta}$ is chosen to be the identity matrix, then the weighting matrix $S$ becomes

$$
S=\left(U^{-1}\right)^{T} U^{-1}=\left[\begin{array}{ll}
\left(Q^{-1}\right)^{\mathrm{T}} Q^{-1} & 0 \\
0 & \left(Q^{-1}\right)^{\mathrm{T}} Q^{-1}
\end{array}\right]
$$

Although it is not analytically proven, we found that the elements of the rows and columns of $S$ associated with the sensitive parts of the structure are larger than those associated with the remaining parts. This is in agreement with the classical design of optimal controllers by LQR in which the magnitudes associated with the displacements, velocities, and control inputs can be regulated by proper selection of their respective diagonal elements in $S$. Therefore, Eq. (21) yields

$$
S_{\eta}=U^{T} S U
$$

The choice of the weighting matrices $S$ and $R$ is a trade-off between control performance ( $S$ large) and low input energy ( $R$ large) [9,19]. They claim that increasing both $S$ and $R$ by the same factor leaves the optimal solution invariant. Their initial guess is to choose both $S$ and $R$ to be diagonal; that is,

$$
\begin{aligned}
& S=\operatorname{diag}\left(S_{1} S_{2} \cdots S_{2 n}\right) \\
& R=\operatorname{diag}\left(R_{1} R_{2} \cdots R_{n}\right)
\end{aligned}
$$

where the $S_{i}$ and $R_{i}$ are positive entries defined by

$$
\sqrt{S_{i}}=\frac{1}{\left|x_{i}\right|^{\max }}
$$

and

$$
\sqrt{R_{i}}=\frac{1}{\left|u_{i}\right|^{\max }} i=1,2, \ldots, 2 n
$$

The quantities $\left|x_{i}\right|^{\max }$ and $\left|u_{i}\right|^{\max }$ denote the maximum absolute acceptable deviation values of the $i^{\text {th }}$ components of the state and control vectors. Both quantities are considered to be the bounds on the states and control inputs. To guarantee the confinement of vibrations, the quantities $\left|x_{i}\right|^{\max }(i=1,2, \ldots, 2 n)$ associated with the sensitive parts must be small resulting in large values of $S_{i}$.

The aforementioned strategy for selecting $R$ and $S$ serves as a design tool for the active control of flexible structures. In such a design, which is considered to be the reference design, performance specifications linked to stability, state and input bounds, and confinement are assumed to be satisfied. In case only passive elements are allowed, we seek an equivalent passive design that uses linear springs and dampers, which leads to a performance similar to that of the active controller. This is will be the subject of the next section.

\section{Design of equivalent passive controllers}

Passive devices produce forces that are proportional to displacement (linear springs), velocity (linear dampers), and/or acceleration (masses). The passive devices employed in this study are limited to linear springs and dampers. Motivated by the work of Gluck et al. [10], we propose the use of the square root of sum of squares (SRSS) method for the design of a passive controller equivalent to the active controller developed in the preceding section. To this end, let the control force vector Eq. (5) be written explicitly as

$$
u=-G x-H \dot{x}
$$


or

$$
\left\{\begin{array}{l}
u_{1} \\
u_{2} \\
\vdots \\
u_{n}
\end{array}\right\}=-\left[\begin{array}{llll}
g_{11} & g_{12} & \ldots & g_{1 n} \\
g_{21} & g_{22} & \ldots & g_{2 n} \\
\vdots & \vdots & \vdots & \vdots \\
g_{n 1} & g_{n 2} & \ldots & g_{n n}
\end{array}\right]\left\{\begin{array}{l}
x_{1} \\
x_{2} \\
\vdots \\
x_{n}
\end{array}\right\}-\left[\begin{array}{cccc}
h_{11} & h_{12} & \ldots & h_{1 n} \\
h_{21} & h_{22} & \ldots & h_{2 n} \\
\vdots & \vdots & \vdots & \vdots \\
h_{n 1} & h_{n 2} & \ldots & h_{n n}
\end{array}\right]\left\{\begin{array}{l}
\dot{x}_{1} \\
\dot{x}_{2} \\
\vdots \\
\dot{x}_{n}
\end{array}\right\}
$$

According to the feedback structure shown in Eq. (27), one can devise a set of interconnected springs and dampers that replaces the active controllers characterized by the feedback gains. The replacement becomes possible only if the feedback matrices are symmetric and tri-diagonal [10]. Therefore, the use of passive elements leads to a new force vector given by

$$
u^{*}(t)=-K_{p} x(t)-C_{p} \dot{x}(t)
$$

or

$$
\left\{\begin{array}{l}
u_{1}^{*} \\
u_{2}^{*} \\
\vdots \\
u_{n}^{*}
\end{array}\right\}=-\left[\begin{array}{llll}
k_{11} & k_{12} & \cdots & k_{1 n} \\
k_{12} & k_{22} & \cdots & k_{2 n} \\
\vdots & \vdots & \ddots & \vdots \\
k_{1 n} & k_{2 n} & \cdots & k_{n n}
\end{array}\right]\left\{\begin{array}{l}
x_{1} \\
x_{2} \\
\vdots \\
x_{n}
\end{array}\right\}-\left[\begin{array}{llll}
c_{11} & c_{12} & \cdots & c_{1 n} \\
c_{12} & c_{22} & \cdots & c_{2 n} \\
\vdots & \vdots & \ddots & \vdots \\
c_{1 n} & c_{2 n} & \cdots & c_{n n}
\end{array}\right]\left\{\begin{array}{l}
\dot{x}_{1} \\
\dot{x}_{2} \\
\vdots \\
\dot{x}_{n}
\end{array}\right\}
$$

The spring and damping constants $k_{i j}$ and $c_{i j}(i, j=1,2, \ldots, 2 n)$ in Eq. (29) can be approximated using the elements of the matrices $G$ and $H$ given in Eq. (27). Next, we outline a scheme for determining a passive control strategy that is equivalent to a given active control structure. Consider the following transformation:

$$
x(t)=T d(t) \quad \text { and } \quad v(t)=T^{T} u(t)
$$

where $T$ is a transformation matrix. Using the above transformation, we rewrite Eq. (30) as

$$
v(t)=-G_{d} d(t)-H_{d} \dot{d}(t)
$$

where

$$
G_{d}=T^{T} G T \quad \text { and } \quad H_{d}=T^{T} H T
$$

Using the same transformation in Eq. (28) yields

$$
v^{*}(t)=-K_{d} d(t)-C_{d} \dot{d}(t)
$$

where

$$
K_{d}=T^{T} K_{p} T=\operatorname{diag}\left(k_{i}\right) \quad \text { and } \quad C_{d}=T^{T} C_{p} T=\operatorname{diag}\left(c_{i}\right)
$$

and $k_{i}$ and $c_{i}$ are supplemental stiffness and damping coefficients associated with the $i^{\text {th }}$ additional passive device.

To determine the individual components of the matrices $K_{d}$ and $C_{d}$ in Eq. (34), we consider the least squares approach. It is assumed that the stiffness and damping matrices $K_{d}$ and $C_{d}$ can be determined independently, and thus the least squares method can be applied separately to approximate each matrix. Using Eqs (32) and (34) and applying the least squares approximation to the difference between Eqs (31) and (33) yields

$$
\begin{aligned}
& \frac{d}{d \dot{d}_{k}}\left\{\int_{0}^{t} \sum_{j}\left[h_{k j} \dot{d}_{j}(\tau)-c_{k} \dot{d}_{k}(\tau)\right]^{2} d \tau\right\}=0 \\
& \frac{d}{d d_{k}}\left\{\int_{0}^{t} \sum_{j}\left[g_{k j} d_{j}(\tau)-k_{k} d_{k}(\tau)\right]^{2} d \tau\right\}=0
\end{aligned}
$$

where $t$ is time for the event considered. The components of the matrices $K_{d}$ and $C_{d}$ can be derived from Eqs (35) and (36). They are given by 


$$
\begin{aligned}
c_{k} & =\int_{0}^{t} \sum_{j} h_{k j} \dot{d}_{j}(\tau) d \tau / \int_{0}^{t} \dot{d}_{k}(\tau) d \tau \\
k_{k} & =\int_{0}^{t} \sum_{j} g_{k j} d_{j}(\tau) d \tau / \int_{0}^{t} d_{k}(\tau) d \tau
\end{aligned}
$$

Assume that at time $t$, the displacement and velocity can be obtained from a modal spectrum approach using the SRSS superposition

$$
\begin{aligned}
& d_{j i}=\left[\sum_{i}\left(\Phi_{j i} P_{i} S_{d i}\right)^{2}\right]^{1 / 2} \\
& \dot{d}_{j i}=\left[\sum_{i}\left(\Phi_{j i} P_{i} S_{v i}\right)^{2}\right]^{1 / 2}
\end{aligned}
$$

where $d_{j i}$ and $\dot{d}_{j i}$ are the displacement and velocity associated with mode $i$ at degree of freedom $j, \Phi_{i j}$ is the differential mass normalized shapes, $P_{i}$ is the participation factor, and $S_{d i}$ and $S_{v i}$ are, respectively, the spectral displacement and velocity of mode $i$. Therefore, approximations of the stiffness and damping constants can be determined from

$$
\begin{gathered}
k_{k}=\frac{\sum_{j} g_{k j}\left[\sum_{i}\left(\Phi_{j i} P_{i} S_{d i}\right)^{2}\right]^{1 / 2}}{\left[\sum_{i}\left(\Phi_{k i} P_{i} S_{d i}\right)^{2}\right]^{1 / 2}} \\
c_{k}=\frac{\sum_{j} h_{k j}\left[\sum_{i}\left(\Phi_{j i} P_{i} S_{v i}\right)^{2}\right]^{1 / 2}}{\left[\sum_{i}\left(\Phi_{k i} P_{i} S_{v i}\right)^{2}\right]^{1 / 2}}
\end{gathered}
$$

In the above approximations, referred to as Full-Mode Approach (FMA), the stiffness and damping are assumed to be independent.

Using the above approximation (FMA), Gluck et al. [10] showed that a second approximation of the damping and stiffness coefficients of supplemental devices in a structure with one dominant mode can be determined from

$$
\begin{gathered}
c_{k}=\frac{\left(\sum_{j} h_{k j} \Phi_{j m}\right)}{\Phi_{k m}} \\
k_{k}=\frac{\left(\sum_{j} g_{k j} \Phi_{j m}\right)}{\Phi_{k m}}
\end{gathered}
$$

Such an approximation is referred to as single-mode approach (SMA).

Furthermore, a third approximation, referred to as single-mode-single-degree approach (SMSDA), can be derived from the SMA by considering a single-gain factor; that is, the one corresponding to degree-of-freedom $k$. Thus, the coefficients of the stiffness and damping can be approximated by

$$
k_{k}=g_{k k}
$$




$$
c_{k}=h_{k k}
$$

This simplified formulation can be obtained directly from Eq. (31) by truncating all of the off-diagonal terms in the matrices $G_{d}$ and $H_{d}$.

The proposed approaches (FMA, SMA and SMSDA) can be used to determine an approximate set of passive elements which if added to the uncontrolled structure yields simultaneous confinement and suppression of vibrations in addition to the regulation of the state and input bounds. It should be pointed out that FMA yields less error between the structural performances resulting from the use of active and passive control strategies. However, if the structure dimension is large, FMA may require an extensive computation time. In this case, one may use either SMA or SMSDA to reduce the computation time at the expense of increasing the performance error. This issue will be addressed in more detail in the next illustrative example.

\section{Illustrative example}

To show the viability of the proposed strategy, we consider the 3DOF oscillator shown Fig. 1. All masses of this oscillator are assumed to be sensitive to vibrations resulting from a base excitation characterized by an acceleration $\ddot{w}(t)$. Since the original oscillator is composed of sensitive parts only, we propose to add inter-mass passive elements that are characterized by inertia, damping, and stiffness. The following configurations are proposed:

1. Configuration 1: one passive element between the first mass and the wall (Fig. 1a).

2. Configuration 2: one passive element between the first mass and the wall and one between the first and second masses (Fig. 1b).

3. Configuration 3: one passive element between the first mass and the wall, one between the first and second masses, and a third one between the second and third masses (Fig. 1c).

The added elements are considered to be the insensitive parts of the system at which the vibration energy is to be confined. The masses, stiffnesses, and damping coefficients of the elements of the oscillator are: $m_{i}=1000 \mathrm{~kg}$, $k_{i}=980 \mathrm{kN} / \mathrm{m}$ and $c_{i}=1.407 \mathrm{kN} \mathrm{sec} / \mathrm{m}(i=1,2,3)$. The mass, stiffness, and damping for all of the passive elements are: $m_{p}=100 \mathrm{~kg}, k_{p}^{\prime}=k_{p}^{\prime \prime}=200 \mathrm{kN} / \mathrm{m}$ and $c_{p}^{\prime}=c_{p}^{\prime \prime}=0.4 \mathrm{kN} \mathrm{sec} / \mathrm{m}$.

Here, we study the performance of the mechanical system subject to an external excitation at the base caused by the 1940 El Centro earthquake scaled to a PGA of $0.112 \mathrm{~g}$. The time response of the uncontrolled system (without passive elements) is shown in Figs $2(\mathrm{a}-\mathrm{c})$ and the base acceleration is displayed in Fig. 2(d). It is clear that the excitation produces large deformations of the oscillator masses. This justifies the need for controllers to reduce vibrations of the sensitive masses.

We first design an active control for each of the aforementioned configurations to confine and suppress the vibratory motion. Every active control strategy uses an appropriate number of force actuators to meet the requirements of confinement and amplitude bounds. Then, we apply the FMA, SMA and SMSDA approaches to determine sets of passive devices (springs and dampers) that replace the active controllers.

The performance of the mechanical system (configuration 1-a) in response to four active forces applied to all masses and the passive element is displayed in Figs 3(a-d) and 4(a-d). It is clear that the vibrations are confined in the added passive element, thereby allowing significant reduction of the vibration amplitudes of the sensitive masses. Tables 1 and 2 summarize the different control parameters and performance indices associated with both of the controlled and uncontrolled responses. In addition, Table 2 demonstrates the performance of the structure for two different forms of the weighting matrix $Q$ associated with configuration 1 . This clearly shows that such weighting has noticeable effect on the vibrational amplitudes and the size of the passive elements. Figure 5 displays the proposed locations of an equivalent set of supplemental passive elements for the purpose of vibration confinement and suppression. Maintaining the active control design the same for all, the use of the FMA, SMA and SMSDA approaches lead to the simulated responses shown in Figs 6(a-d), 7(a-d) and 8(a-d). This set of figures indicates that the FMA approach yields a more accurate replacement of the forces with the added passive elements (compare Figs 3 and 6). The other approaches, although there are performance errors as compared with the active control, lead to simultaneous confinement and suppression of vibrations. Table 3 gives the performances resulting from the use of the FMA, SMA and SMSDA approaches as compared with that of the actively controlled system. The supplemental 


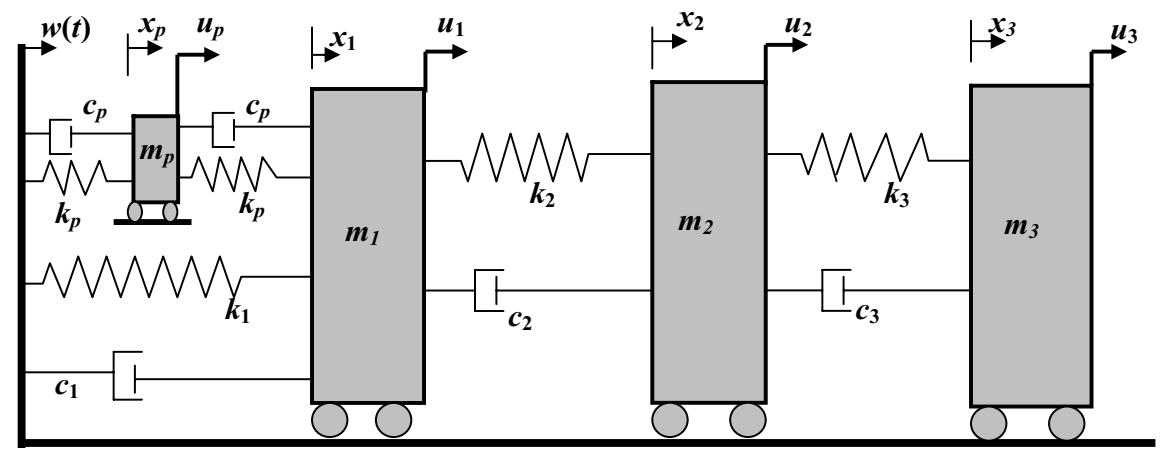

(a)

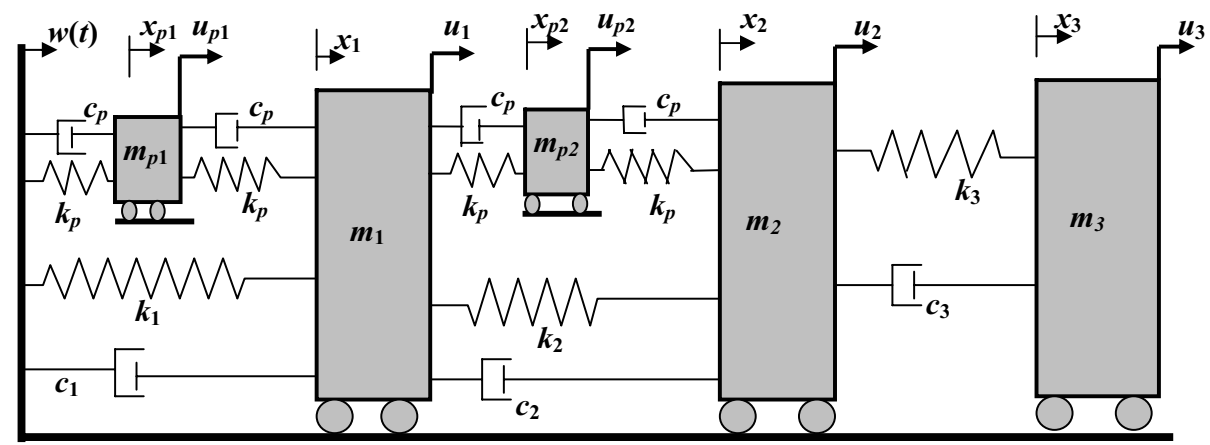

(b)

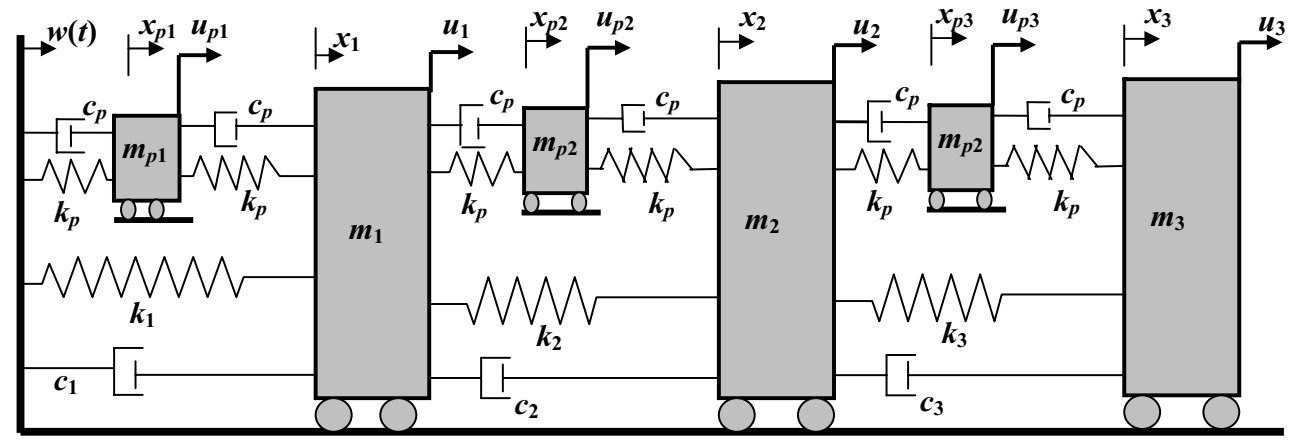

(c)

Fig. 1. (a) Three DOF oscillator with one passive element (configuration 1); (b) Three DOF oscillator with two passive elements (configuration 2); (c) Three DOF oscillator with three passive elements (configuration 3).

stiffness and damping matrices $K_{d}$ and $C_{d}$ associated with the three approaches are displayed in Table 4. Note that the use of FMA leads to lower values of stiffness and damping coefficients. This constitutes an advantage in the sense that adding passive elements with low stiffness and damping coefficients is more appealing as far as implementation is concerned.

To show the effectiveness of reducing the demand on the supplemental stiffness and damping matrices $K_{d}$ and $C_{d}$, we consider the use of the FMA, SMA and SMSDA approaches for oscillator configurations 2 and 3 (see Figs 1-b and 1-c). Table 5 summarizes the resulting stiffness and damping matrices $K_{d}$ and $C_{d}$. In addition, Table 6 displays the performances resulting from the use of the FMA, SMA and SMSDA approaches, associated with both configurations as compared with that of the actively control systems. 
(a)

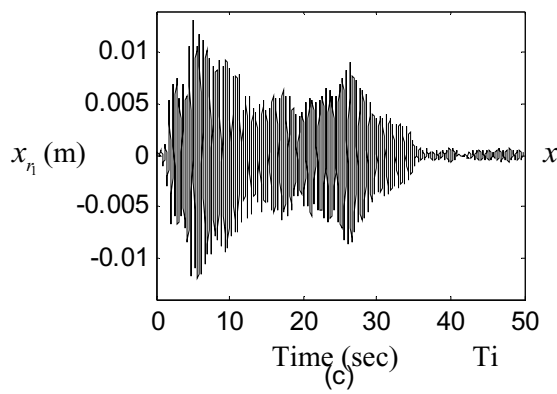

(b)
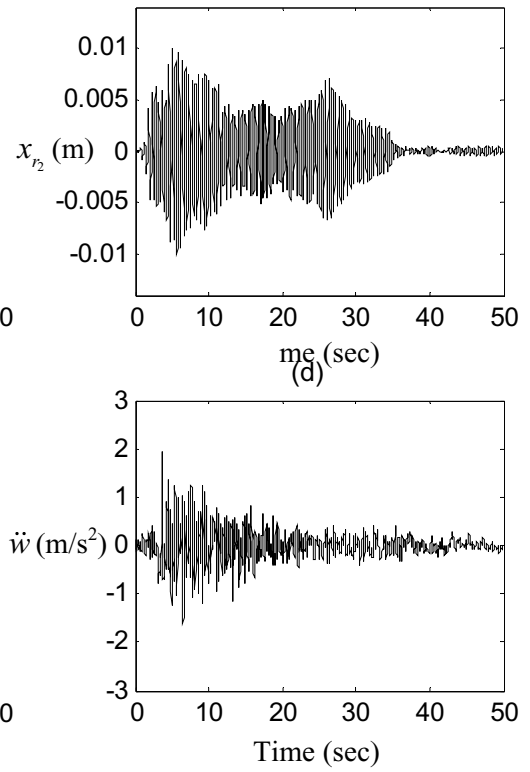

Fig. 2. Relative displacements of the uncontrolled system. (a) First mass $x_{r 1}=x_{1}$; (b) Second mass $x_{r 2}=x_{2}-x_{1}$; (c) Third mass $x_{r 3}=x_{3}-x_{2}$; (d) Base acceleration.
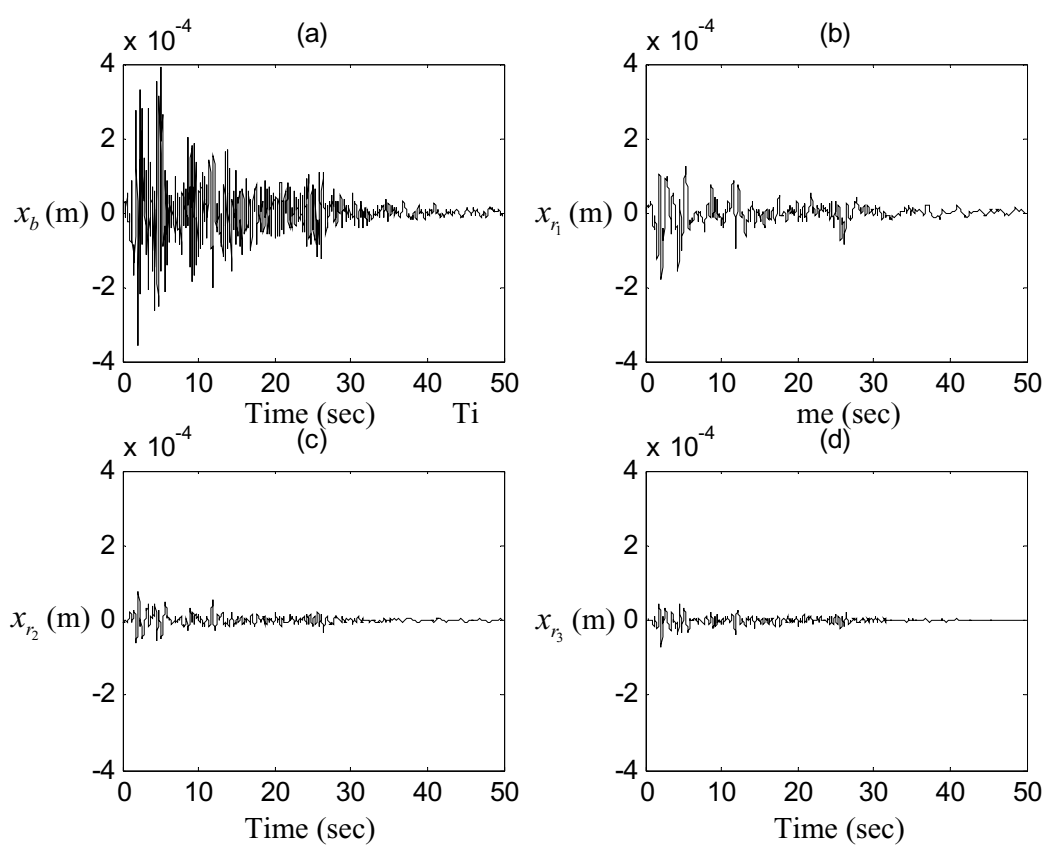

Fig. 3. Relative displacements associated with the LQR-controlled response. (a) Passive element $x_{r 1}=x_{p}$; (b) First mass $x_{r 2}=x_{1}$; (c) Second mass $x_{r 3}=x_{2}-x_{1}$; (d) Third mass $x_{r 4}=x_{3}-x_{2}$.

We note that Choura [5] showed that control efforts, which confine and suppress the vibratory motion and use magnitudes equal to those applied in classical control strategies such as the pole allocation method, yield improved structural performance. Of course, lowering the magnitudes of these control efforts is feasible with possible performance degradation. This degradation can be small or high depending on how much the magnitudes are 
Table 1

Weighting and confinement matrices

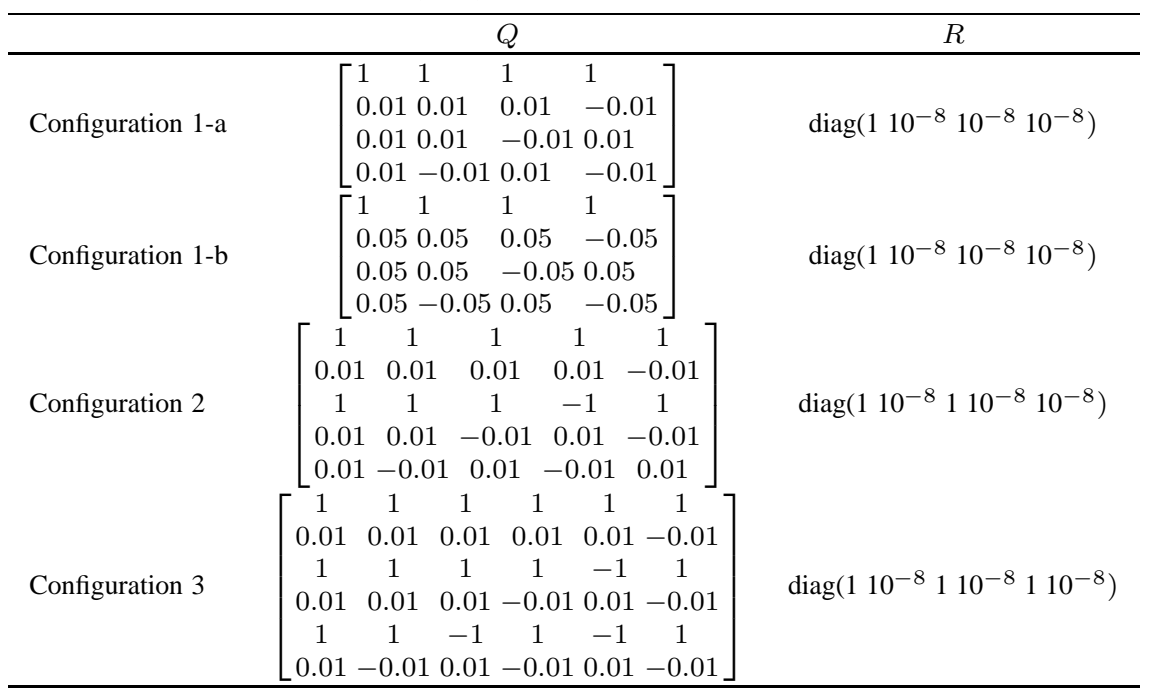
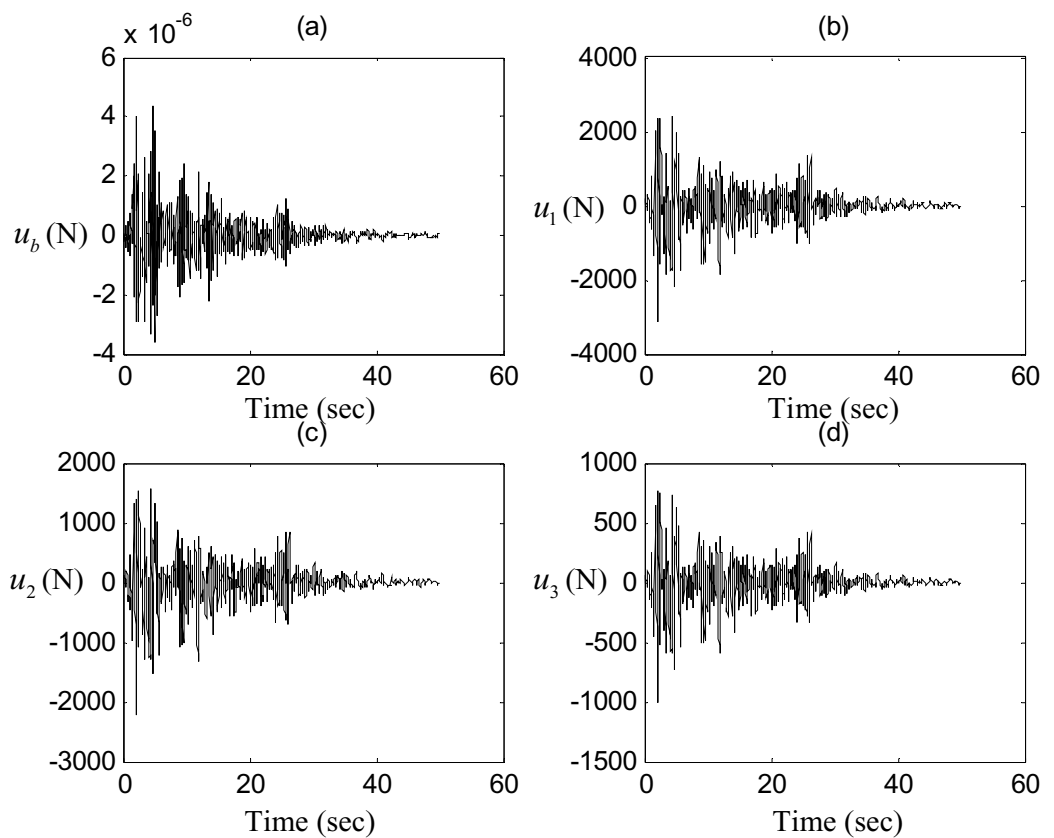

Fig. 4. Force simulations of active LQR-controlled structure by confinement. (a) Passive element $u_{r}=u_{p}$; (b) First mass $u_{r 2}=u_{1}$; (c) Second mass $u_{r 3}=u_{2}-u_{1}$; (d) Third mass $u_{r 4}=u_{3}-u_{2}$.

lowered. With regard to the passive approach, the reduction of the mass/stiffness of the added elements is of great importance. It should be noted that the mass/stiffness of these elements can be determined once the active controller is specified via knowledge of the matrices $Q$ and $R$. For the illustrative example at hand, it was verified that reducing the mass/stiffness can be attained by lowering the magnitudes of $Q$ and $R$. 
Table 2

Different parameters and performance indices of the controlled and uncontrolled systems

\begin{tabular}{llcccc}
\hline & & Mass 1 & Mass 2 & Mass 3 & $\begin{array}{c}\text { Passive } \\
\text { element }\end{array}$ \\
\hline No control & Max relative displacement $(\mathrm{mm})$ & 13 & 10 & 5.8 & - \\
& Max acceleration $\left(\mathrm{cm}^{2} \mathrm{~s}^{2}\right)$ & 305 & 465 & 570 & - \\
Active control & Max relative displacement $(\mathrm{mm})$ & 0.18 & 0.08 & 0.07 & 0.39 \\
(Configuration 1-a) $)$ & Max acceleration $\left(\mathrm{cm} / \mathrm{s}^{2}\right)$ & 108 & 108 & 108 & 158 \\
& Max applied force $(\mathrm{kN})$ & 3.14 & 2.22 & 1.01 & $4.3710^{-6}$ \\
Active control & Max relative displacement $(\mathrm{mm})$ & 0.63 & 0.24 & 0.09 & 0.57 \\
(Configuration 1-b) & Max acceleration $\left(\mathrm{cm} / \mathrm{s}^{2}\right)$ & 108 & 107 & 107 & 154 \\
& Max applied force $(\mathrm{kN})$ & 2.73 & 2.23 & 1.03 & $5.910^{-6}$ \\
\hline
\end{tabular}

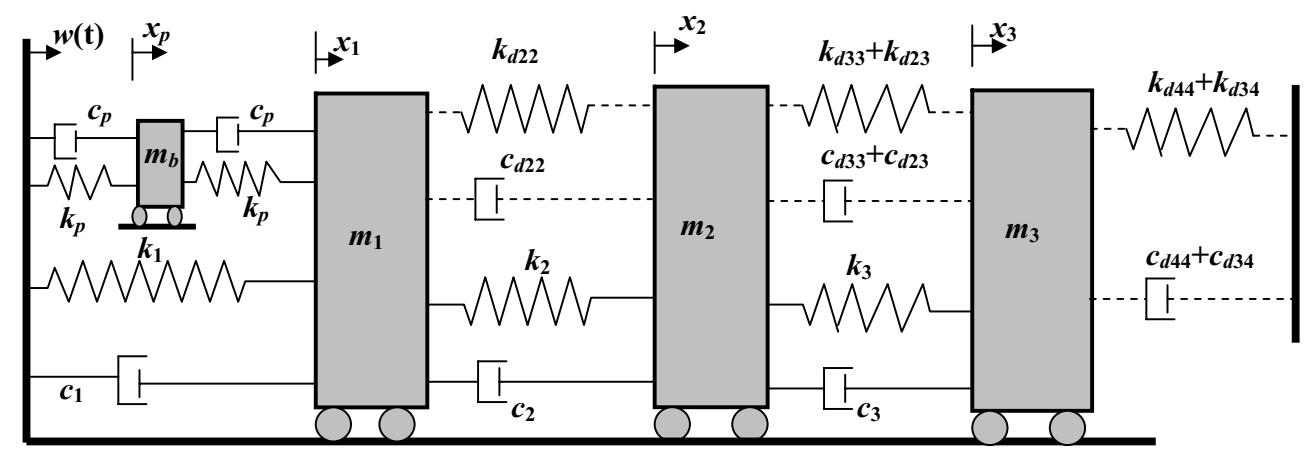

Fig. 5. Oscillator with the supplemental springs and dampers.
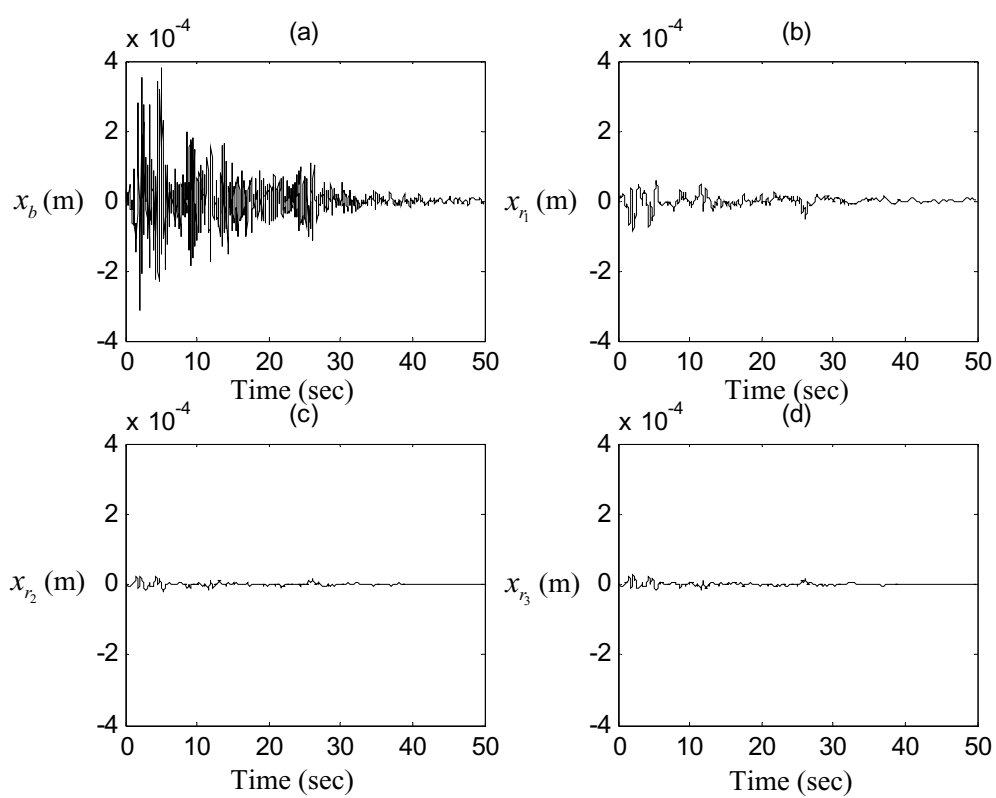

Fig. 6. Relative displacement of passive controlled structure by confinement with the FMA approach. (a) Passive element $x_{r} 1=x_{p}$; (b) First mass $x_{r 2}=x_{1}$; (c) Second mass $x_{r 3}=x_{2}-x_{1}$; (d) Third mass $x_{r 4}=x_{3}-x_{2}$.

\section{Conclusion}

A design strategy for the simultaneous confinement and suppression of vibrations in flexible structures by using passive devices is presented. The proposed design first synthesizes a reference control law using feedback. It is 
Table 3

Configuration 1-performance resulting from the use of the FMA, SMA and SMSDA approaches

\begin{tabular}{llcccc}
\hline & & Active control & FMA & SMA & SMSDA \\
\hline Max relative & Passive element & 0.394 & 0.382 & 0.385 & 0.459 \\
displacement (mm) & First mass & 0.181 & 0.083 & 0.177 & 0.404 \\
& Second mass & 0.079 & 0.024 & 0.029 & 0.127 \\
& Third mass & 0.072 & 0.025 & 0.056 & 0.134 \\
Acceleration & $\max \left|\ddot{x}_{p}\right|$ & 158.34 & 158.64 & 157.72 & 154.16 \\
$\left(\mathrm{~cm} / \mathrm{s}^{2}\right)$ & $\max \left|\ddot{x}_{1}\right|$ & 107.74 & 107.69 & 107.57 & 107.80 \\
& $\max \left|\ddot{x}_{2}\right|$ & 107.53 & 107.74 & 107.61 & 107.60 \\
& $\max \left|\ddot{x}_{3}\right|$ & 107.69 & 107.81 & 107.71 & 107.69 \\
\hline
\end{tabular}
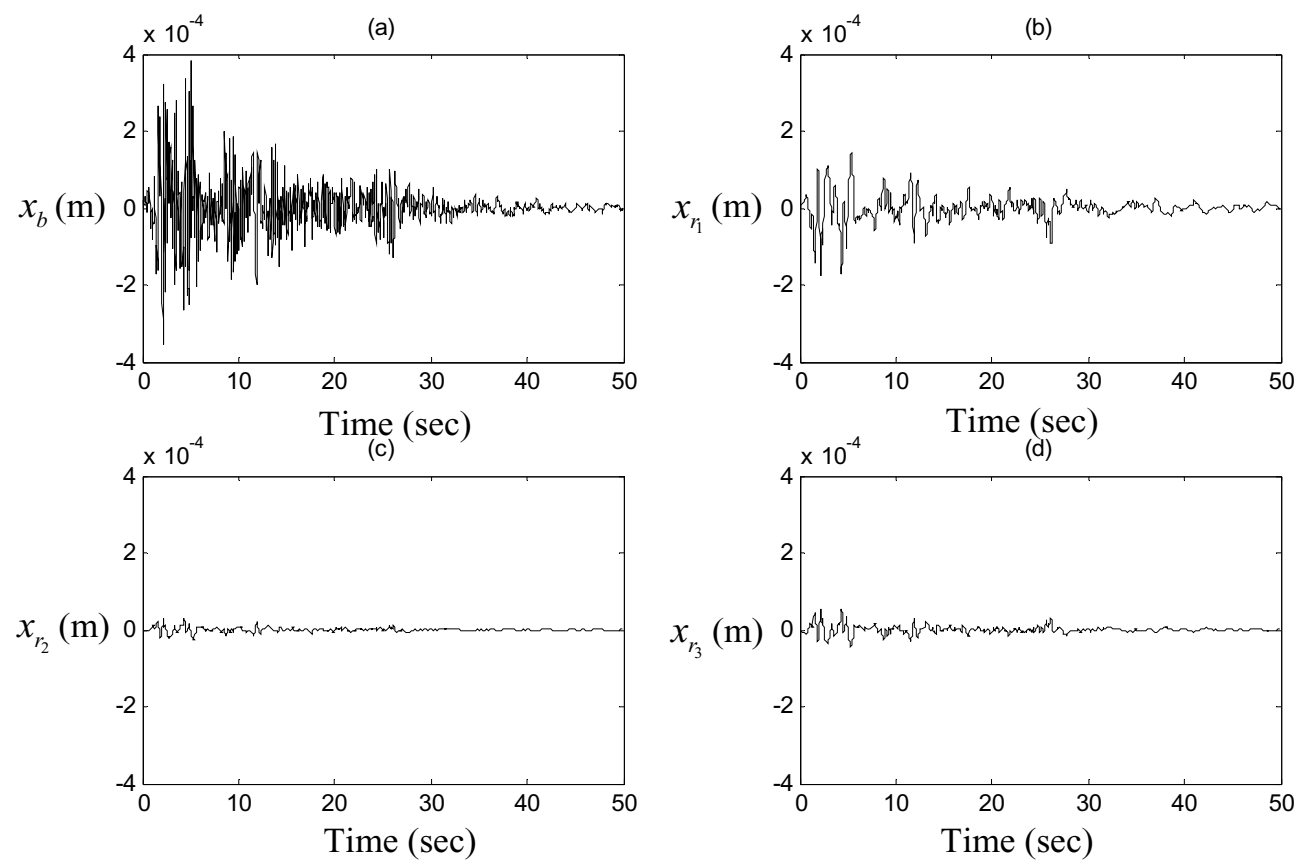

Fig. 7. Relative displacement simulations of passive controlled system by confinement with the SMA approach. (a) Passive element $x_{i} 1=x_{p}$; (b) First mass $x_{r 2}=x_{1}$; (c) Second mass $x_{r 3}=x_{2}-x_{1}$; (d) Third mass $x_{r 4}=x_{3}-x_{2}$.

demonstrated that the problem of SCSV can be formulated as an LQR-optimal control problem. Such a formulation allows the regulation of the maximum amplitudes associated with the control input and the displacements of the sensitive and insensitive parts. A transformation technique that yields an equivalent passive controller is then proposed. This technique uses the SRSS method to determine an approximate equivalent passive controller that maximizes the effects of added passive elements. We demonstrated the efficiency of the proposed control design using a 3DOF mechanical oscillator subject to an external excitation. All of the masses of the oscillator are assumed to be sensitive to the excitation, and thus the vibratory energy is confined in the added passive elements. It was shown that the vibration amplitudes associated with the masses of the oscillator are attenuated at fast rate at the expense of slowing down the convergence of the passive elements to their steady states. Similar structural performances resulting from the use of the RCL and the equivalent passive control strategy are found for different configurations of the modified system.

Future research will address the issue of applying the proposed control strategy to real-world flexible structures. Modifying existing structures by adding passive elements to suppress and confine vibrations would be of great importance in civil engineering structures. For instance, the proposed strategy can be applied to historical buildings that suffer serious degradations and cracks. 

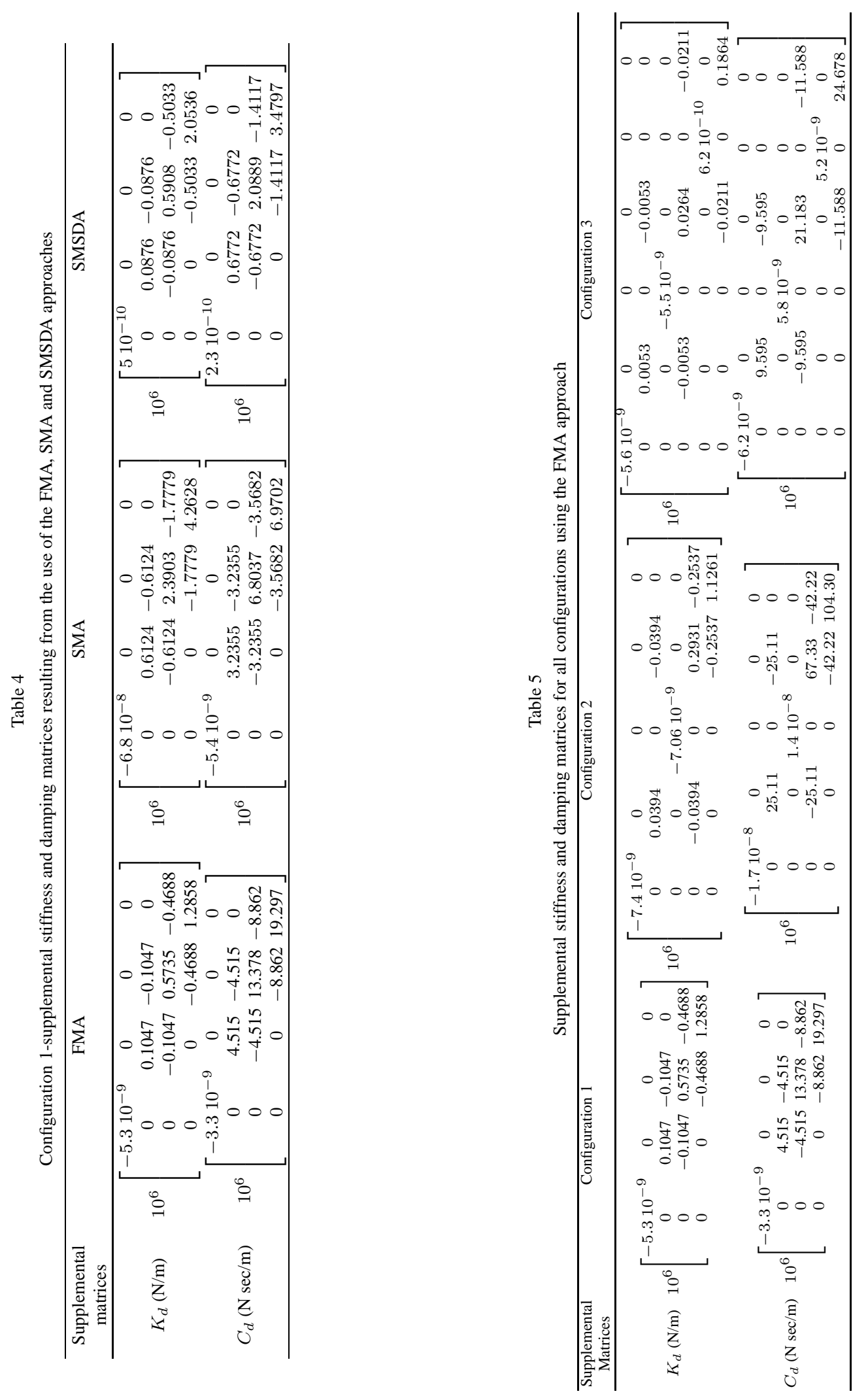


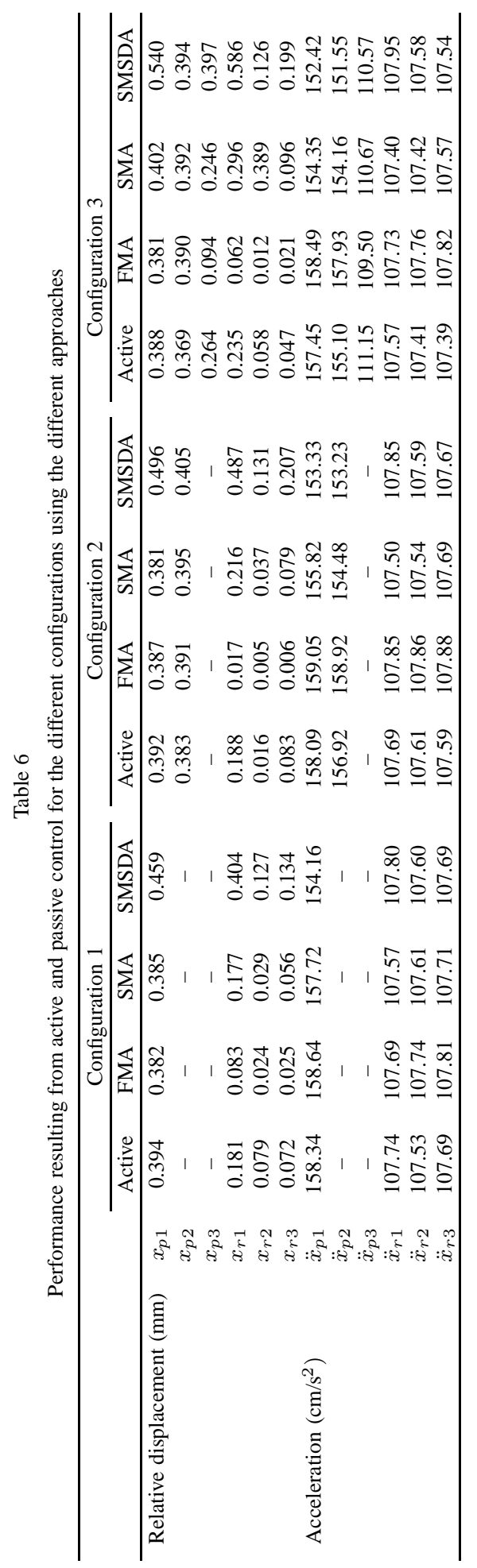



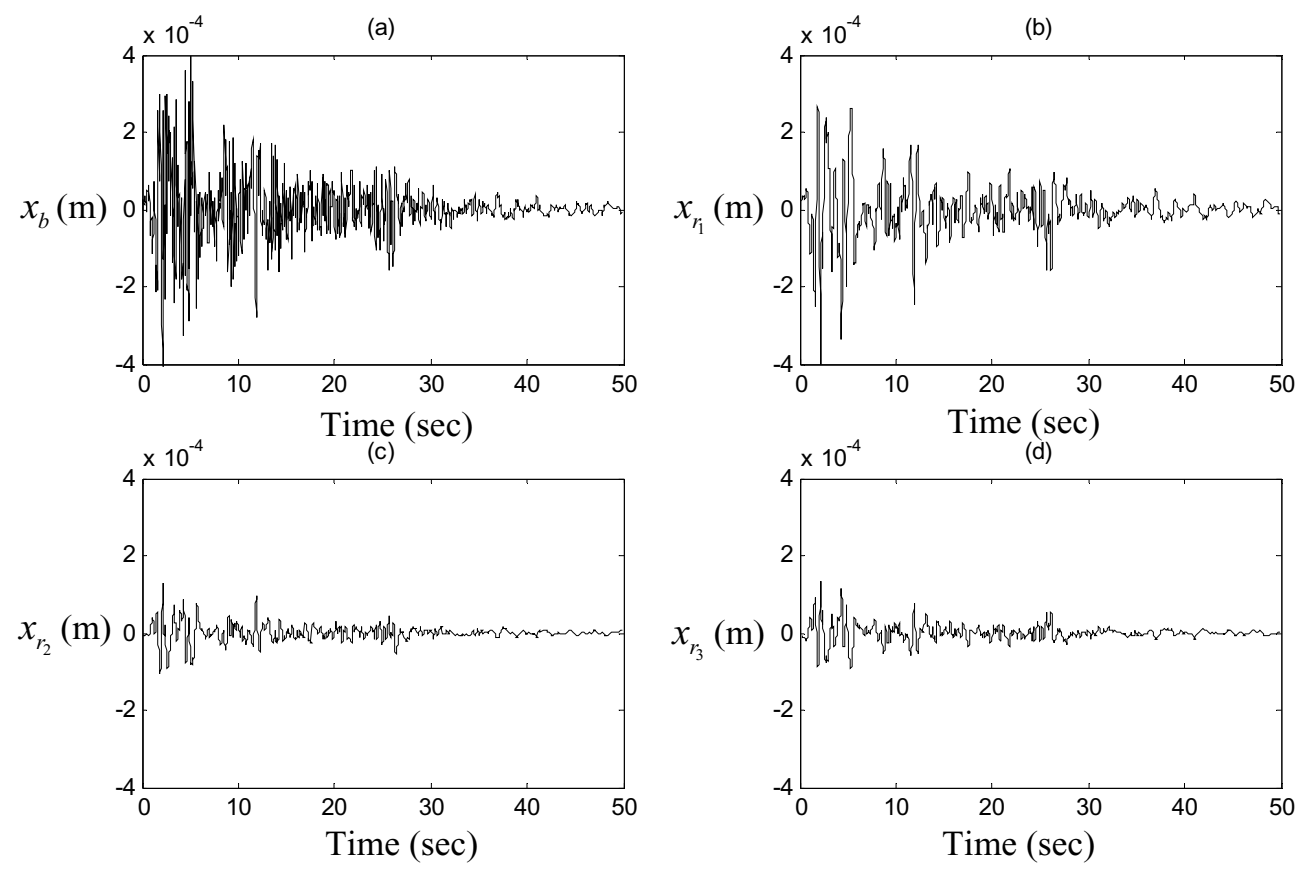

Fig. 8. Relative displacement simulations of passive controlled system by confinement with the SMSDA approach. (a) Passive element $x_{r} 1=x_{p}$; (b) First mass $x_{r 2}=x_{1}$; (c) Second mass $x_{r 3}=x_{2}-x_{1}$; (d) Third mass $x_{r 4}=x_{3}-x_{2}$.

\section{References}

[1] O.O. Bendiksen, Localization Phenomena in Structural Dynamics, Chaos Solitons and Fractals 11 (2000), $1621-1660$.

[2] M.P. Castanier and C. Pierre, Lyapunov Exponents and Localization and Dissipation Phenomena in Multi-Coupled Nearly Periodic Systems, Journal of Sound and Vibration 168(3) (1993), 479-505.

[3] M.P. Castanier and C. Pierre, Individual and Interactive Mechanisms for Localization and Dissipation in a Mono-Coupled Nearly-Periodic Structure, Journal of Sound and Vibration 183(3) (1995), 493-515.

[4] J.W. Choi and Y.B. Seo, LQR Design with Eigenstructure Assignment Capability, IEEE Transactions on Aerospace and Electronic Systems 35(2) (1999), 700-708.

[5] S. Choura, Control of Flexible Structures with the Confinement of Vibrations, ASME Journal of Dynamic Systems, Measurement, and Control, USA 117(2) (1995), 155-164.

[6] S. Choura and A.S. Yigit, Vibration Confinement in Flexible Structures by Distributed Feedback, Journal of Computers and Structures 54(3) (1995), 531-540.

[7] S. Choura and A.S. Yigit, Confinement and Suppression of Structural Vibrations, ASME Journal of Vibration and Acoustics 123 (2001), 496-501.

[8] S. Choura, S. El-Borgi, A. Abichou and A. Nayfeh, Control of Seismically Excited Structures by Passive Confinement of Vibrations, in the 3rd Word Conference on Structural Control, Como, Italy, April 2002, 7-11.

[9] H.J. Coelingh, T.J.A. Vries, P.B.A. Lochem and J. Amerongen, Automated Optimal Controller Design in Mechatronics, 7th IFAC Symp. on Computer Aided Control Systems Design (CACSD’97), Gent, Belgium, 1997.

[10] N. Gluck, A.M. Reinhorn, J. Gluck and R. Levy, Design of Supplemental Dampers for Structures, ASCE Journal of Structural Engineering 122(12) (1996), 1394-1399.

[11] R.S. Langley, On the Forced Response of One-Dimensional Periodic Structures: Vibration Localization by Damping, Journal of Sound and Vibration 178(3) (1994), 411-428.

[12] T. Kobori and S. Kamagata, Dynamic Intelligent Buildings-Active Seismic Response Control, in: Intelligent Structures-2, Y.K. Wen, ed., Elsevier Applied Science, New York, 1992, pp. 279-282.

[13] M. Ouled Chtiba, S. Choura, S. El-Borgi and A.H. Nayfeh, Hybrid Control of Seismically Excited Structures by Vibration Confinement, 13th World Conference on Earthquake Engineering Vancouver, B.C., Canada, August 1-6, 2004, n.1860.

[14] K. Seto, A. Ooshima and H. Fujii, Active Vibration Control of a Bridge Tower Structure using Filtered LQ Control Technique, the 4th International Conference on Motion and Vibration Control, Yokohama, Japan, 1998.

[15] K.A. Snyder and T.R. Kirkpatrick, The Influence of Anderson Localization on the Mode Decay of Excited Non-Linear Systems, Annelen dur Physik (Leipzig) 8 (1999), SI-241-244, Gaithersburg, MD 20899 USA.

[16] A.M. Reinhorn, N. Gluck, J. Gluck and R. Levy, Optimal Design of Supplemental Dampers for Control of Structures, 11th European Conference in Earthquake Engineering, Paris, 1998. 
[17] A.M. Reinhorn, N. Gluck, J. Gluck and R. Levy, Optimal Design Dampers and Stiffeners in Structures using Active Control Approaches, Third European Conference on Structural Control, Paris, 2000.

[18] Y. Ribakov, J. Gluck and N. Gluck, Practical Design of MDOF Structures with Supplemental Viscous Dampers Using Mechanical Levers, 8th ASCE Specialty Conference on Probabilistic Mechanics and Structural Reliability, PMC2000-005, 2000.

[19] I. Robandi, K. Nishimori, R. Nishimura and N. Ishihara, Optimal Feedback Control Design Using Genetic Algorithm in Multi-Machine Power System, Electrical Power \& Energy Systems 23 (2001), 263-271.

[20] S.M. Shahruz, Elimination of Vibration Localization in Mistuned Periodic Structures, Journal of Sound and Vibration 281 (2005), 452-462.

[21] S.M. Shahruz, Elimination of Vibration Localization: a Mathematical Justification, Journal of Sound and Vibration 283 (2005), 449-458.

[22] K.V. Singh and Y.M. Ram, Dynamic Absorption by Passive and Active Control, Journal of Vibration and Acoustics 122 (2000), 1-5.

[23] A.S. Yigit and S. Choura, Vibration Confinement in Flexible Structures via Alteration of Mode Shapes Using Feedback, Journal of Sound and Vibration 179 (1995), 553-567. 

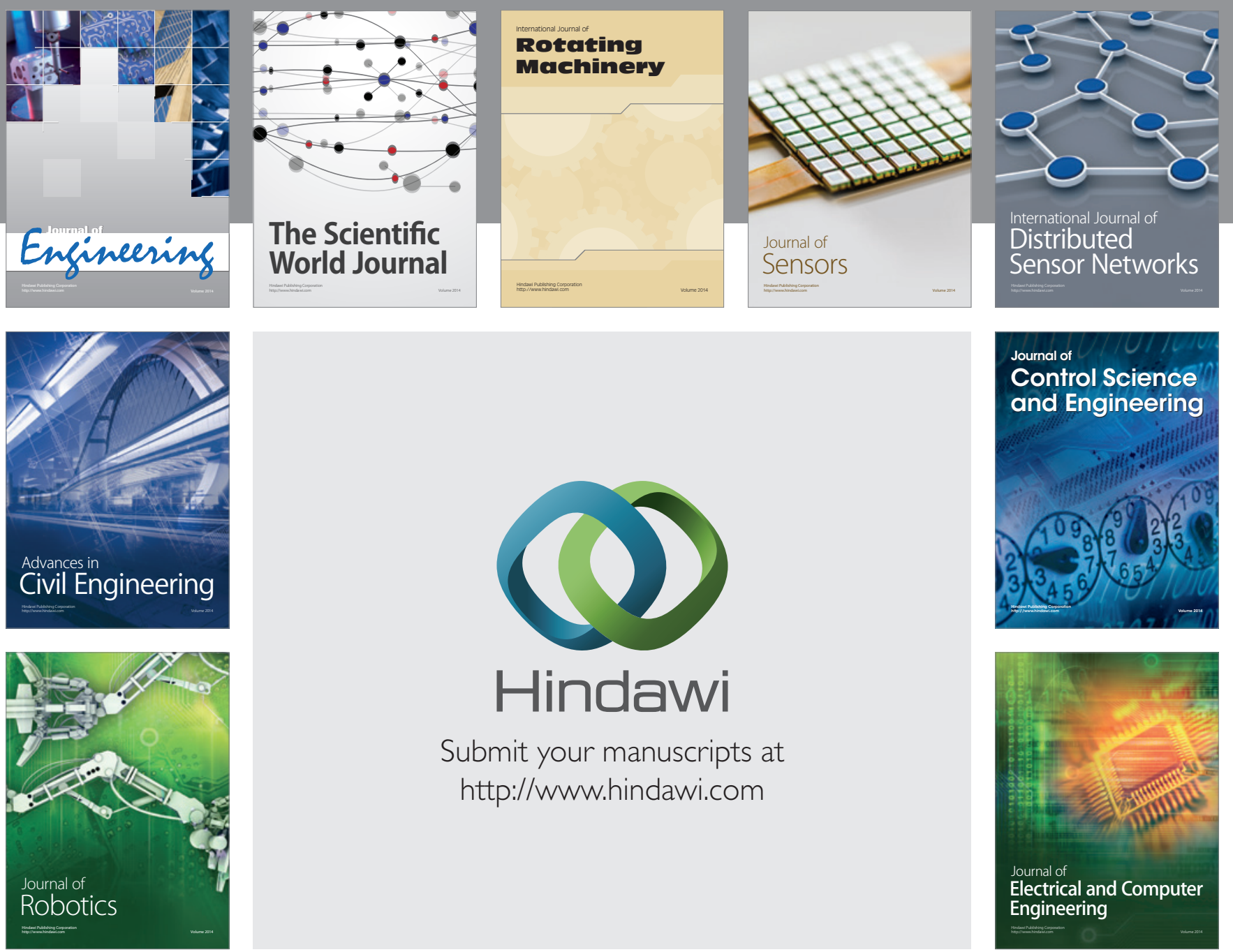

Submit your manuscripts at

http://www.hindawi.com
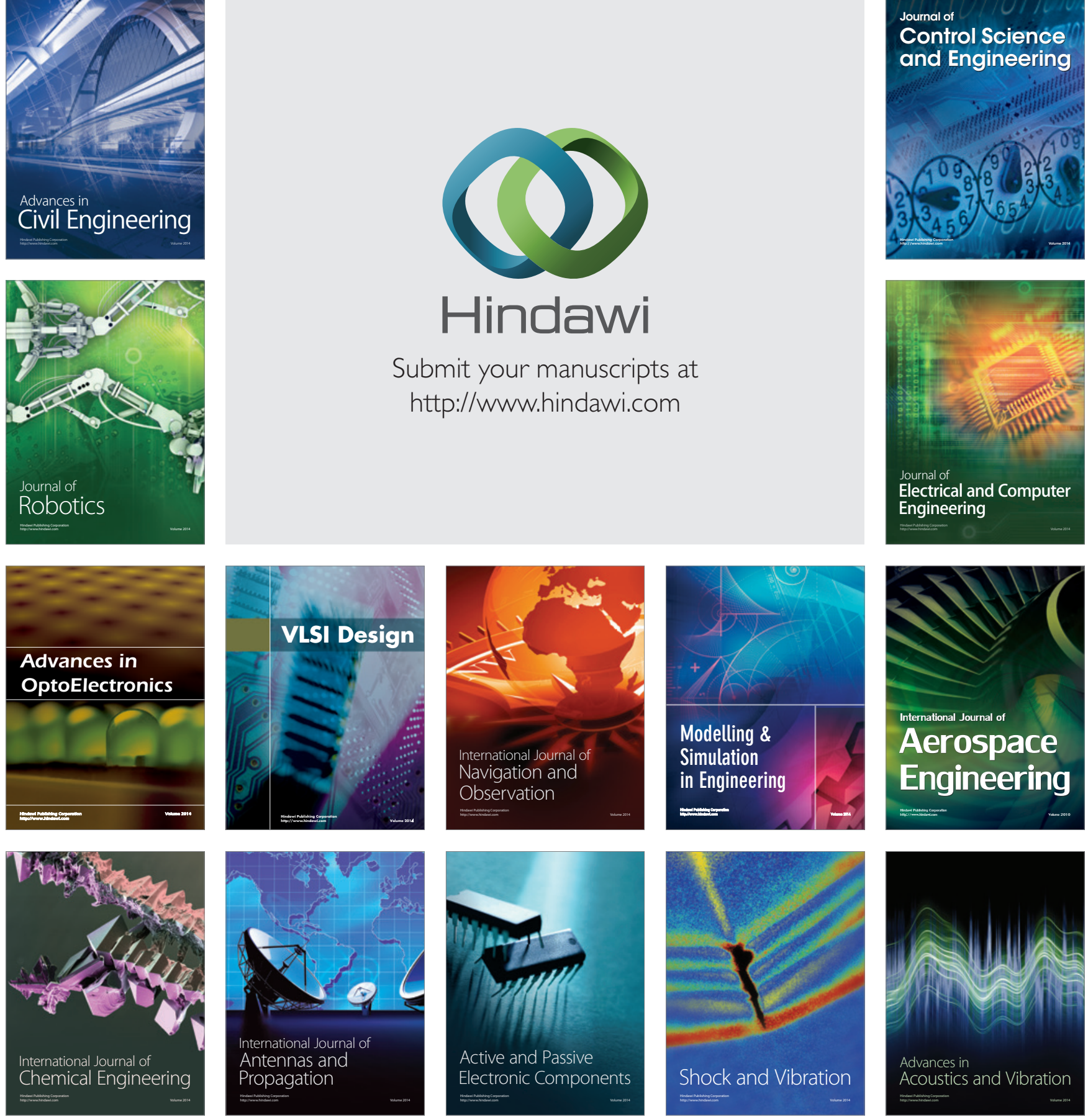